Supplement of Biogeosciences Discuss., 11, 8789-8828, 2014

http://www.biogeosciences-discuss.net/bgd-11-8789-2014/

doi:10.5194/bgd-11-8789-2014-supplement

(C) Author(s) 2014. CC Attribution 3.0 License.

(c) (i)

Supplement of

\title{
Quantifying legacies of clearcut on carbon fluxes and biomass carbon stock in northern temperate forests
}

W. Wang et al.

Correspondence to: W. Wang (wang.weifeng@unh.edu) 
1 Table S1 Parameter values used in the simulations of PnET-CN for both deciduous broadleaf

2 forests (DBF) and evergreen needleleaf forests (ENF) in Upper Midwest region of Wisconsin

3 and Michigan, United States.

\begin{tabular}{|c|c|c|c|}
\hline Parameter & Description & DBF & ENF \\
\hline \multicolumn{4}{|c|}{ Canopy and photosynthesis } \\
\hline AmaxA & Intercept (A) and slope (B) for relationship & -46 & 5.3 \\
\hline AmaxB & $\begin{array}{l}\text { between Foliar } \mathrm{N} \text { and max. photosynthesis (nmol } \\
\mathrm{CO}_{2} \mathrm{~g}^{-1} \text { leaf } \mathrm{s}^{-1} \text { ) }\end{array}$ & 71.9 & 21.5 \\
\hline AmaxFrac & $\begin{array}{l}\text { Daily Amax as a fraction of early morning } \\
\text { instantaneous rate }\end{array}$ & 0.76 & 0.76 \\
\hline CFracBiomass & Carbon fraction of biomass & 0.49 & 0.49 \\
\hline DVPD1 & \multirow{2}{*}{$\begin{array}{l}\text { Coefficients for power function converting VPD to } \\
\text { fractional lose in photosynthesis }\end{array}$} & 0.05 & 0.05 \\
\hline DVPD2 & & 2 & 2 \\
\hline FolRelGroMax & $\begin{array}{l}\text { Maximum relative growth rate for foliage (\% year } \\
1 \text { ) }\end{array}$ & 0.95 & 0.3 \\
\hline FolReten & Foliage retention time (year) & 1 & 2.3 \\
\hline HalfSat & Half saturation light level $\left(\mathrm{J} \mathrm{m}^{-2} \mathrm{~s}^{-1}\right)$ & $150^{\mathrm{a}}$ & $150^{\mathrm{a}}$ \\
\hline $\mathrm{k}$ & Canopy light attenuation constant coefficient & 0.58 & $0.48^{\mathrm{a}}$ \\
\hline PsnTMin & Minimum temperature for photosynthesis $\left({ }^{\circ} \mathrm{C}\right)$ & 4 & 0 \\
\hline PsnTopt & Optimum temperature for photosynthesis $\left({ }^{\circ} \mathrm{C}\right)$ & 24 & 22 \\
\hline SLWDel & $\begin{array}{l}\text { Change in specific leaf weight (SLW) with } \\
\text { increasing foliar mass above }\left(\mathrm{g} \mathrm{dm} \mathrm{m}^{-2} \mathrm{~g}^{-1}\right)\end{array}$ & 0.2 & 0 \\
\hline SLWMax & Top canopy SLW (g dm m²) & $81^{\mathrm{a}}$ & $258^{\mathrm{a}}$ \\
\hline \multicolumn{4}{|l|}{ Respiration } \\
\hline Ksom & $\begin{array}{l}\text { Decomposition rate for soil organic carbon pool } \\
\left(\text { year }^{-1}\right)\end{array}$ & 0.075 & 0.075 \\
\hline BaseFolRespFrac & $\begin{array}{l}\text { Dark respiration as a fraction of max. } \\
\text { photosynthesis }\end{array}$ & $0.05^{\mathrm{b}}$ & $0.05^{b}$ \\
\hline GRespFrac & $\begin{array}{l}\text { Ratio of growth respiration to component } \\
\text { production }\end{array}$ & 0.20 & 0.20 \\
\hline RootMRespFrac & $\begin{array}{l}\text { Ratio of fine root maintenance respiration to root } \\
\text { biomass production }\end{array}$ & 1 & 1 \\
\hline RespQ10 & $\mathrm{Q}_{10}$ value for leaf maintenance respiration & $2.5^{\mathrm{c}}$ & $2.5^{\mathrm{d}}$ \\
\hline WoodMRespA & $\begin{array}{l}\text { Fraction of wood maintenance respiration over } \\
\text { total photosynthesis. }\end{array}$ & 0.07 & 0.07 \\
\hline
\end{tabular}




\begin{tabular}{|c|c|c|c|}
\hline \multicolumn{4}{|l|}{ Allocation } \\
\hline PlantCReserveFrac & $\begin{array}{l}\text { Fraction of non-structural plant } \mathrm{C} \text { held in reserve } \\
\text { after allocation to wood carbon }\end{array}$ & 0.75 & 0.75 \\
\hline RootAllocA & \multirow{2}{*}{$\begin{array}{l}\text { Intercept (A) and Slope (B) of relationship } \\
\text { between foliar and root allocation }\end{array}$} & 0 & 0 \\
\hline RootAllocB & & 2 & 2 \\
\hline MinWoodFolRatio & $\begin{array}{l}\text { Minimum ratio of carbon allocation to wood and } \\
\text { foliage }\end{array}$ & 0.8 & 0.5 \\
\hline \multicolumn{4}{|l|}{ Turnover } \\
\hline WoodLitCLose & $\begin{array}{l}\text { Fractional loss of woody mass as } \mathrm{CO}_{2} \text { in wood } \\
\text { decomposition }\end{array}$ & 0.8 & 0.8 \\
\hline WoodLitLoseRate & $\begin{array}{l}\text { Fractional loss of woody mass either to soil } \\
\text { organic carbon pool or to atmosphere }\end{array}$ & 0.1 & 0.1 \\
\hline WoodTurnover & Fractional mortality of live wood per year & 0.025 & 0.02 \\
\hline RootTurnoverA & \multirow{3}{*}{ Coefficients for fine root turnover } & 0.789 & 0.789 \\
\hline RootTurnoverB & & 0.191 & 0.191 \\
\hline RootTurnoverC & & 0.021 & 0.021 \\
\hline \multicolumn{4}{|l|}{ Phenology } \\
\hline GDDFolEnd & $\begin{array}{l}\text { Growing degree days of at which foliage } \\
\text { production ends }\left({ }^{\circ} \mathrm{C}\right)\end{array}$ & 764 & 1031 \\
\hline GDDFolStart & $\begin{array}{l}\text { Growing degree days of at which foliage } \\
\text { production onset }\left({ }^{\circ} \mathrm{C}\right)\end{array}$ & 332 & 332 \\
\hline GDDWoodEnd & $\begin{array}{l}\text { Growing degree days of at which wood production } \\
\text { ends }\left({ }^{\circ} \mathrm{C}\right)\end{array}$ & 764 & 1031 \\
\hline GDDWoodStart & $\begin{array}{l}\text { Growing degree days of at which wood production } \\
\text { onset }\left({ }^{\circ} \mathrm{C}\right)\end{array}$ & 332 & 332 \\
\hline SenescStart & Day of year after which leaf drop can occur (days) & 270 & 270 \\
\hline \multicolumn{4}{|l|}{ Nitrogen $(N)$} \\
\hline FLPctN & Minimum N concentration in foliar litter (\%) & 0.9 & 0.45 \\
\hline FolNConRange & Maximum fractional increase in $\mathrm{N}$ concentrations & 0.5 & 0.7 \\
\hline FolNRetrans & Foliage N retention time (year) & 0.5 & 0.5 \\
\hline MaxNStore & $\begin{array}{l}\text { Maximum N content in non-structural N pool (g N } \\
\mathrm{m}^{-2} \text { ) }\end{array}$ & 76 & 76 \\
\hline NImmobB & \multirow{2}{*}{$\begin{array}{l}\text { Coefficients for fraction of mineralized } \mathrm{N} \\
\text { remobilized as a function of soil organic matter }\end{array}$} & 151 & 151 \\
\hline NImmobA & & -35 & -35 \\
\hline RLPctN & Minimum N concentration in root litter (\%) & 1.2 & 1.2 \\
\hline
\end{tabular}




\begin{tabular}{llcc}
\hline WLPctN & Minimum N concentration in wood litter (\%) & 0.2 & 0.2 \\
Water balance & & & \\
$\mathrm{f}$ & Soil water release parameter & 0.04 & 0.04 \\
FastFlowFrac & Fraction of water inputs lost directly to drainage & 0.1 & 0.1 \\
PrecIntFrac & $\begin{array}{l}\text { Fraction of precipitation intercepted and } \\
\text { evaporated }\end{array}$ & 0.11 & 0.11 \\
WUEConst & $\begin{array}{l}\text { Water use efficiency at } 1 \mathrm{kPa} \text { of vapor pressure } \\
\left.\text { deficit (g CO2 } \mathrm{kg}^{-1} \mathrm{H}_{2} \mathrm{O}\right)\end{array}$ & 10.9 & 10.9 \\
\hline
\end{tabular}

$1 \quad{ }^{\mathrm{a}}$ Estimated based on measurements of Ryu et al. (2008).

$2{ }^{\mathrm{b}}$ Estimated based on estimated percentage (4.5-9.7\%) of leaf respiration to total ecosystem

3 respiration by Bolstad et al. (2004).

$4 \quad{ }^{\mathrm{c}}$ Estimated based on $\mathrm{Q}_{10}$ range (2.27-2.98) from measured leaf respiration rates of Bolstad et al.

5 (2004).

$6 \quad$ d Estimated based on the synthesis study of Atkin \& Tjoelker (2003). 

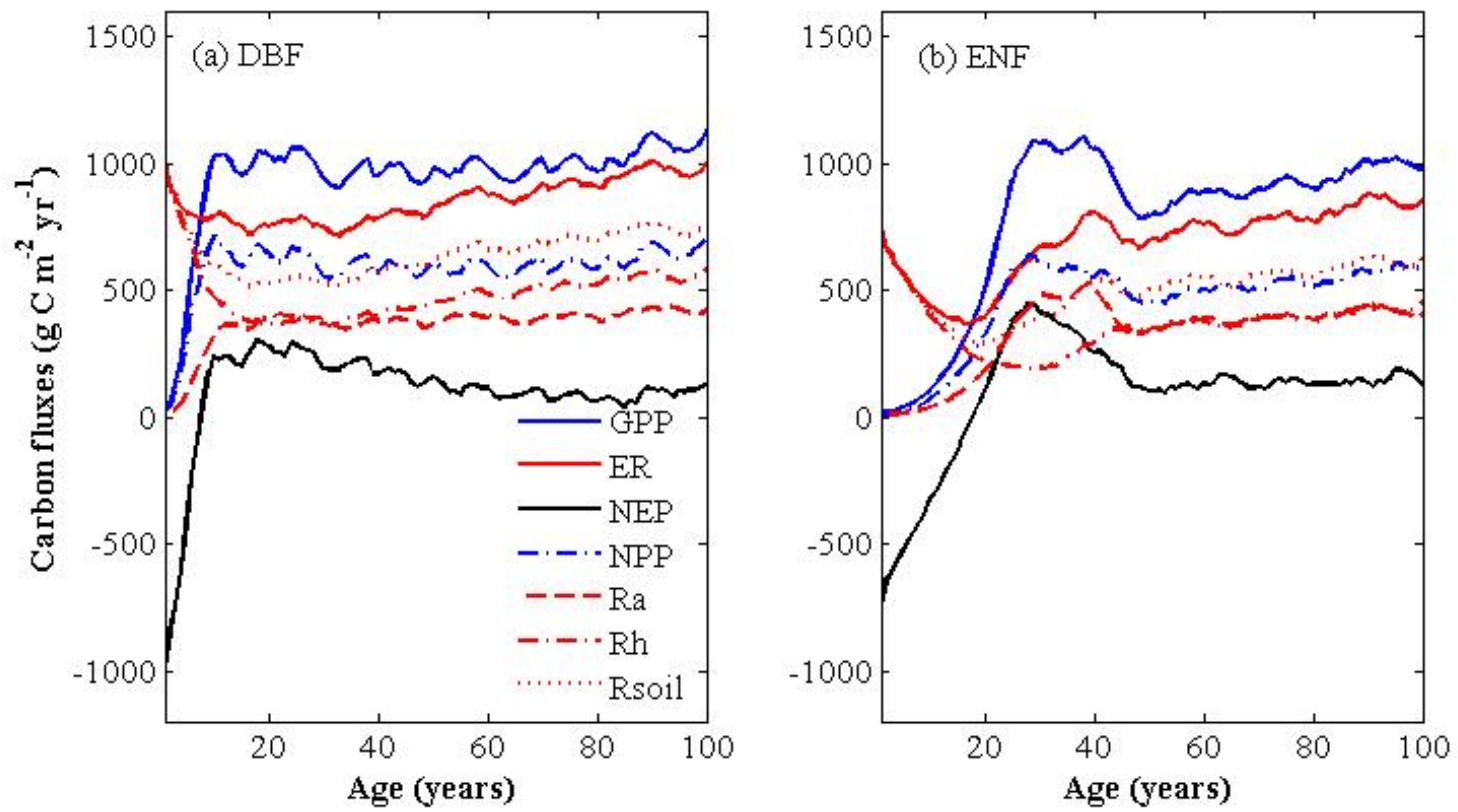

2 Figure S1. Simulated trajectories of ecosystem carbon fluxes (GPP, gross primary production;

3 ER, ecosystem respiration; NEP, net ecosystem production; NPP, net primary production; Ra,

4 autotrophic respiration; Rh, heterotrophic respiration; Rsoil, soil respiration) with stand age for

5 (a) deciduous broadleaf forests (DBF) and (b) evergreen coniferous forests (ENF). The simulated

6 curves were smoothed using a moving average filter with a span of 5. 


\section{References:}

2 Atkin, O.K. and Tjoelker, M.G., 2003. Thermal acclimation and the dynamic response of plant respiration to temperature. Trends Plant Sci., 8(7): 343-351.

4 Bolstad, P.V., Davis, K.J., Martin, J., Cook, B.D. and Wang, W., 2004. Component and whole5 system respiration fluxes in northern deciduous forests. Tree Physiol., 24(5): 493-504.

6 Ryu, S.-R., Chen, J., Noormets, A., Bresee, M.K. and Ollinger, S.V., 2008. Comparisons 7 between PnET-Day and eddy covariance based gross ecosystem production in two

8 Northern Wisconsin forests. Agric. For. Meteorol., 148(2): 247-256. 